\title{
Moving in the right direction
}

New data published in a recent issue of Molecular Microbiology might have finally solved the controversy over the function of SpoIIIE during sporulation in Bacillus subtilis.

During sporulation, B. subtilis undergoes an asymmetric cell division that generates two cells of unequal size - a small forespore and a larger mother cell - and a chromosome must be accurately segregated into each cell. The asymmetric-division septum is formed before chromosome segregation is completed, pinching one of the replicated chromosomes into a small lobe, which constitutes $\sim 30 \%$ of the genome and is present in the forespore, and a large lobe, which constitutes $\sim 70 \%$ of the genome and is present in the mother cell. To ensure successful completion of chromosome segregation, the trapped large lobe must be translocated across the septum from the mother cell into the forespore.

Chromosome segregation during sporulation requires the doublestranded DNA translocase SpoIIIE, a protein that is related to the FtsK translocase of non-spore-forming bacteria. In recent years, results from different laboratories have supported two models of SpoIIIE/FtsK function. In the first, which is based on single-molecule studies of FtsK, the proteins function as reversible DNA translocases, and the polarity of the translocated DNA is determined by the DNA substrate. In the second, SpoIIIE functions as a DNA exporter, and the polarity of the translocated DNA is determined by the cellspecific assembly of a stable SpoIIIE

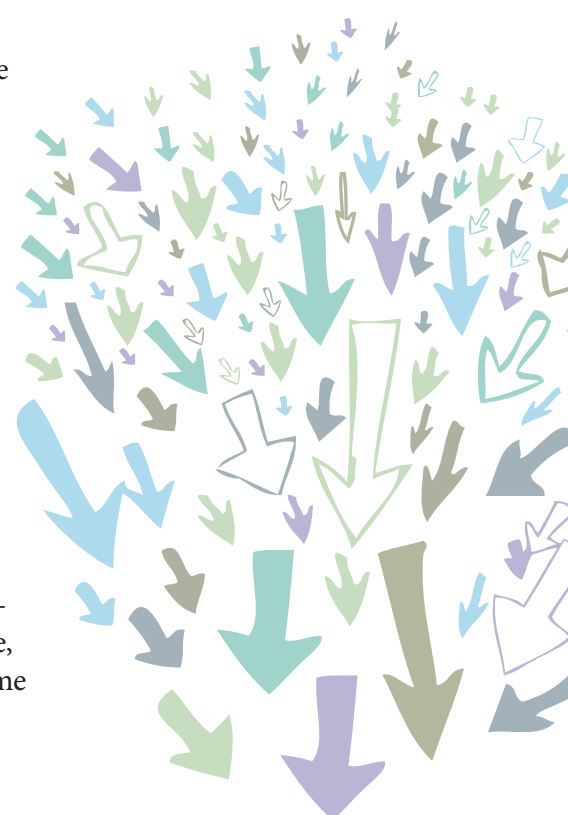

translocation

complex. In this

work, Eric Becker and Kit Pogliano

set out to determine which of these models is correct.

They began by devising a method that would allow them to tag SpoIIIE specifically in the mother cell and the forespore. To do so, the authors made use of the high-affinity interaction between the leucine zipper domains of cFos and cJun - the leucine zipper domain of cFos was fused to the carboxyl terminus of SpoIIIE and the leucine zipper domain of cJun was fused to the amino terminus of green fluorescent protein (GFP). Cellspecific promoters were used to express this GFP fusion protein in either the mother cell or the forespore. The results showed that wild-type SpoIIIE forms a focus only in the mother cell, supporting the second model discussed above. However, analysis of translocation-defective SpoIIIE showed that, in the absence of DNA translocation, SpoIIIE complexes formed on both sides of the septum, supporting the first model.

To resolve this conundrum, Becker and

Pogliano moved on to look at the effects of chromosome orientation. In a B. subtilis strain in which the chromosome partitioning proteins Soj and Spo0J had been deleted, the SpoIIIE complex could assemble in, and move DNA out of, the forespore. Finally, using a range of time-lapse fluorescence microscopy studies the authors were able to show that there was a direct correlation between the polarity of SpoIIIE-mediated chromosome segregation and the position of oriC after septation - SpoIIIE moves the chromosome into the cell that contains the replication origin.

So, it seems that aspects of both models of SpoIIIE/FtsK function were correct - the assembly of a stable SpoIIIE translocation complex is indeed cell-specific, but this specificity, and the resulting translocation polarity, are determined by the orientation of the chromosome. Sheilagh Molloy

ORIGINAL RESEARCH PAPER Becker, E. C. \& Pogliano, K. Cell-specific SpollIE assembly and DNA translocation polarity are dictated by chromosome orientation. Mol. Microbiol. 66 1066-1079 (2007) 